\title{
Impact of Research or Research on Impact: More Than a Matter of Semantics and Sequence
}

\author{
Julian May and Roxana Barrantes
}

\section{Introduction}

The rich data and analysis contained in this volume permit a number of lessons to be drawn, some of which resolve the questions identified by the authors and others which may inspire new inquiries. Looking at the variety of topics, disciplines and areas of research, a palpable conclusion is that ICT now pervades the lives of people throughout the world. This is unsurprising since ICTs are general purpose technologies, widely adopted, used and adapted by people of all ages worldwide and who are from different socioeconomic backgrounds. By focusing on the global south, however, one outstanding characteristic of the research relates to the different pace of use and appropriation within these countries. This is apparent in both the theoretical chapters and the evidence gathered from the empirical ones. Whatever the pace, it is apparent that ICT is having an impact on most economic sectors and social processes. This impact may be positive or negative and is not independent of the context into which ICT is inserted or the existing socioeconomic dynamics of that context.

In the concluding chapter to an earlier edited volume, Harris and Chib (2012) propose three possible areas through which research may have influence: capacity development, socioeconomic benefits and policy impact. We add a fourth and suggest that the lessons drawn from the research contained in this book also

J. May, Ph.D. (ه)

Institute for Social Development, University of the Western Cape, Bellville, Western Cape,

South Africa

e-mail: jmay@uwc.ac.za

R. Barrantes, Ph.D.

Instituto de Estudios Peruanos, Lima, Peru

e-mail: roxbarrantes@iep.org.pe

A. Chib et al. (eds.), Impact of Information Society Research in the Global South, DOI 10.1007/978-981-287-381-1_15 
contribute towards theory and, in some instances, reveal areas in which further theory building is required. In this chapter we will focus on policy and theory impacts.

In the realm of policy influence, more than simply building appropriate linkages to policymakers, the media and development practitioners is required. The timing and content of these linkages must be considered and better understanding is needed of the politics and process of policy. Without this, significant resources may be invested in research but without proper communication, and engagement with multiple stakeholders, lessons arising from the gathered evidence may be lost, limited to circles with no real influence into either policy or theory, or misused. This is true of any topic and the case of ICT is no exception.

In the case of theory, it is apparent that the studies in this volume fall into two groups. There are those which find that ICT amplifies existing structures and dynamics as is proposed by Agre (2002) and more recently Toyama (2011), and thus, existing theory may need only extension. There are those in which ICT reconfigures existing structures and dynamics as proposed by Dutton (2004) and supported by interventionists such as Brewer et al. (2005). In these cases, existing theory may be inadequate, may be out of date or does not exist.

\section{Research on Impact}

The objective for many of the chapters in this volume is identifying the evidence concerning the impact of ICT on society and its transformation. The volume begins to collect and draw together evidence about the impact that ICTs are having and, as such, contributes new findings to the already vast body of knowledge that informs about how ICTs are contributing to society. In this respect, it is generally recognized that there are both social and economic dimensions of change and, further, that consideration should be given to both actual and perceived changes in deprivation. This is a substantial question that requires a more sustained research effort than the one undertaken by the SIRCA II research cycle. As the chapter by Flor reminds us, ICT can have an impact on sectors (such as agriculture, health or education) and on development themes (such as poverty, gender or governance). The mechanisms through which this impact takes place are complex, as are the sectors and themes in which the impact is to be felt. In addition, information and communications is a fast-growing economic sector in its own right and directly contributes towards the growth of a quaternary sector of knowledge-based services.

Attribution of change to ICT, whether positive or not, is thus complex and links of causation may flow in different directions. As with any attribution attempt, it is necessary to separate outputs, outcomes and impacts and to decide which is feasible to measure, as well as which is necessary before conclusions can be reached concerning the contribution of the intervention. Thought must also be given as to what is meant by 'causation' and whether it can be convincingly demonstrated. There are also lags between ICT interventions and their impact, and these lags in 
turn may result in confounding influences that further test the ability to determine causation and the mechanisms through which change is brought about. Finally, as Flor observes, there are unintended consequences to which any intervention is prone. All this should be expected for as policy analyst Ray Pawson (2006:35) comments, interventions are 'complex systems thrust amidst complex systems'. Further, in these systems they compete with prior, existing and new interventions for impact.

The field of ICTD possibly has an advantage over other forms of research in development studies as it is relatively specific about the link to be investigated between the intervention and its intended outcome. By keeping people communicated and informed, the underlying expectation is that society will be strengthened in some way and individuals will not only be better off but will feel better as well. Taking advantage of this, an important step that is consciously made in several of the chapters attempting to trace the link from an ICT activity to change is the deconstruction of how such links manifest.

Questioning how an ICT activity manifests, several authors in this volume have proposed options. As an example, Dodel and Ramirez emphasize a triad: access, use and appropriation. Thus, for ICT to bring benefits to individuals, households, communities and society in general, ICT must be accessible, used and appropriated - meaning that users both adopt and adapt ICT to meet their needs. This then can increase well-being (following a mainly economics perspective) or augment capabilities and freedoms (following a Sen-oriented framework). Also in this volume, Diga and May speak of access, ownership and usage. Elsewhere Heeks (2010) has proposed a similar triad of readiness, availability and uptake. In each case ICT cannot simply be present in order to have an impact and must satisfy additional criteria of usage.

For the sake of brevity, we will focus our discussion of research on impact in the following domains: education, gender and society, poverty and political participation. Regarding education, useful evidence is provided from Peru, where achievement is very low representing a deep developmental problem. Through participatory action research (PAR) the team set out to adapt pedagogical tools to online platforms, working directly with school teachers. These were initially disconcerted when confronted with the technologies, but their confidence improved along their computational skills. An important lesson that comes out of the study is that a key interaction that should be taken into consideration when designing educational materials is the one between teachers and software developers.

Two studies inform our conclusions on gender and society, both from Asia. Being in the forefront of IS development is an aspiration shared by many countries, the Philippines being not exception. We learned that legislation may not keep up when there is a clash between how ICTs are used for a living, i.e. cybersex, and society's efforts to fight cybercrime. Understood as affective labour, cybersex challenges moral-based perceptions about crime and poses the question as to why it should be penalized, especially in a context in which women have few decent employment alternatives. 
We have evidence from Indonesia showing how effective social media can be to boost women's entrepreneurship abilities and outcomes because of the flexibilities it offers. In turn, this percolates positively into women's position in the family and household. Women may feel empowered due to their social media use.

Diga and May's review of the literature suggests that evidence is leaning towards ICT on average reducing poverty rather than exacerbating it. This work shows that ICT access among poor people is now high in many countries and is boosted by shared ownership of handsets and in some instance SIM cards. Numerous applications now exist for mobile phones which are tailored towards the needs of the poor, with ways of transferring money conveniently and at low cost being one of the most frequent uses. They comment on the high willingness of poor people to commit resources to ICT which can run at around $25 \%$ of income and they show that the poor spend a greater share of their income on ICT than the nonpoor. This could be a significant drain on household resources if this usage does not result in income generation or cost reductions elsewhere. This is not necessarily impoverishment since information and communication may bring about greater freedoms in other dimensions of well-being. Alternatively it could mean improvements in the quality of life of some within a household at the cost of others and may have particular gendered consequences. More direct measurement of well-being would help resolve this, perhaps through indicators such as those used for happiness studies might be an option.

Other chapters have traced the link between ICT and well-being. The case study in the Ivory Coast reveals that mobile phones are no panacea since they are used by people already engaged in specific social and power relations, as is the case with any other ICT. We learn that women take advantage of the mobile for reasons similar to those heard of in other places: security, reduced coordination costs, avoided transportation costs and so forth. But we also learn that some people may inhibit use due to their traditional beliefs, some of which contribute towards a fear of the technology.

Regarding political influence, chapters in the volume have revealed that ICT can affect both politics and policies and, equally, that ICT can affect governance and not simply government. These terms are often used interchangeably in the literature, including in this volume, but the distinction is important. Policies might not be an issue, their proper implementation may well be. Supporting this view, ICT is often blamed, by repressive governments, or thanked, by community organizations, for enabling social contact for political mobilization. Two research projects, one from Brazil and the other from China, show this. The Brazilian study focuses on political consultations processes aided by ICT, which is a promising tool to encourage participation and strengthen democratic processes as a by-product. Different results are found depending on which platform is used, the requisites that each one demands and the functionalities allowed, particularly taking into consideration the stage in the consultation process in which the Web 2.0 tool is used. For instance, in the initial stages of agenda setting, platforms allowing for comments and social interaction may be recommended. 
In China, where migrant workers face a whole set of hurdles to legally work outside their place of origin, through ICT, the intention of expression of young migrant workers is considered high, beginning with interpersonal communication in social media, up to institutional channels, which are less personal. This finding is important since young people find a way of expression, through ICT, in an otherwise closed society, facing a difficult predicament because of migration. What is apparent from these case studies is that it is not appropriate to view either ICT or (D)evelopment as being apolitical and instead ICT is embedded in society/polity.

\section{Impact of Research}

Several authors emphasize that impact of research is a process which begins when the research project is designed. This has resonance with the chapter by Harris and could be separated into the intent to influence, the means to influence, the opportunity to do, the absorptive capacity of those being influenced, their willingness to be influenced, their ability to influence others in power and the extent to which influence can endure into the creation or reform of policy and the implementation of policy. In considering this, several authors note that when all relevant stakeholders are engaged from the onset, research and communication are better linked, and the results informed by policy considerations and as a result are rapidly taken into consideration in policymaking. This demands different attitudes and skills from researchers as well as a new way to schedule research activities. Not only mastering research methodologies and being at the forefront of research methods are in order, but the ability to communicate and convey ideas in lay terms. Further, these processes of moving research findings into policy do not occur in a vacuum but in the institutional framework in each country or subnational partition. These may be functioning, well established and integrated, but can be partial, politicized and disconnected.

This is further complicated by the modes and protocols of communication that are appropriate to the sectors being investigated. For example, the chapters show how work on educational issues and professionals is different than from that which involves interacting with health specialists. In this volume, this issue is illustrated by the discussion on how ICT affects the different logics of health care: choice or care. The case teaches us the importance of an open mind when designing and implementing interventions and the necessity to regard the cultural dimensions of the people involved, that is, those whose well-being is supposed to increase by the intervention.

Wider issues concerning policy impact are commented on by several of the authors who problematize the question. These authors question what policy can be influenced by research, when and under what circumstances as well as the extent to which such influence is desirable. Furthermore, governments are by no means the most important sites of decision-making in ICT in which a relatively small number of large multinational companies provide services globally. As such, 
the priorities of different stakeholders will differ, and those of the private service providers accountable to international shareholders are sure to differ from those of governments accountable to their citizens and local businesses.

Addressing this is tricky. As is pointed out by Ordóñez in this volume, if research is to be useful, it must be timely. This means that appropriate research questions may have to be determined in advance of their need by policymakers. Policymakers are under pressure to deliver and cannot necessarily wait for the gestation and delivery of rigorous research. Thus, time must be allowed for data collection, analysis and interpretation and the development of appropriate communication strategies that feed into the policy cycle of government. The point of intervention into the policy cycle is by no means clear and will vary both by the policy under review and according to the structures of governance and over time. Further, over the medium to long term, there are distinct policymaking episodes when governments may be more open to research.

Addressing these issues is not without challenge. Funding is not necessarily available for as yet unfelt needs, while researchers are not necessarily integrated into governance structures and may not know the appropriate points through which to enter the policy dialogue. In addition, most researchers must also manage their own academic cycles and obligations, and these may conflict with those of the government. As a result it should also not be taken as a given that policymakers are the only or prime audience of researchers. There are other users of research which researchers may wish to prioritize, including their peers and the users of ICT themselves. An interesting pathway for research influence is implicit in the discussion by Steibel and Estevez on ICT and public participation. Thus, researchers who wish to be activists in taking forward their findings could use such processes to bring their findings to the attention of both citizens and policymakers.

Once these issues have been resolved, the results must be wisely communicated but in a manner that is nonetheless accessible to the audience, whether decision makers or beneficiaries. Pathways to influence risk becoming oversimplified to marketing exercises and the development of packages which make research results appear palatable. There is also the concern that research findings may be presented as being more conclusive than the data actually permit or that only research questions which lend themselves to apparently conclusive results are deemed worthy of policy influence.

These challenges are well documented elsewhere (e.g. Boaz and Pawson 2005) and it is important that donors of research funding realize that longer research cycles may be needed in order to deal with these complexities. These should specifically include a component which focuses on building pathways to submit results into debates over policy and its implementation. In some cases, this is already recognized and a few grant-making bodies now require that reporting on research output and impact extends well beyond the period that has been funded. The United Kingdom's Economic and Social Research Council (ESRC) is an example.

Turning now to the impact of research on theory building, ICT4D is a relatively new topic and various contributions in the book address the novelties associated. At the same time, the chapters have demonstrated opportunities to use existing theory 
in application to ICT with a diverse theoretical base drawn from Habermas, Spinoza, Bourdieu and Putnam. Theory development also offers a way of resolving some of the difficulty of attribution mentioned above. By developing and testing theories of change that consider how, why and under what circumstance an anticipated impact of ICT may take place, attribution can at least be deduced if not measured.

The chapters in the book deal with a variety of approaches of development, from which several concepts are used - and these are not necessarily interchangeable. From the mainstream MDG discussion, and the need to build theories of change along the unintended consequences of ICT interventions, to the discussion around the inextricably linked relationships between ICT and poverty, the first part is mainly theoretical, dwelling with relevant concepts and methodological approaches to include ICT as a part of answers to development challenges. In addition, much is made of putting the D back into ICTD. But ' $D$ ' has always been a contested notion. ICTD is equally of relevance in wealthy countries in which there is significant poverty, and development is a concern in wealthy countries which are consuming unsustainable quantities of the world's non-renewable assets. The ICTD debate also should begin to separate out what is development from what is development studies, the latter being a field of critical inquiry concerning the contemporary dynamics of social, economic, political and population change that makes use of interdisciplinary conceptual frameworks and methods to identify development destinations that are possible and desirable, for whom and at what cost. In addition, theory building in this respect should distinguish between the study of ICT for developing countries and the study of ICT in developing countries.

The notion that communication can bring about developmental change is not new (e.g. Stevenson 1988). The notion that differential access to information may impact on economic development is somewhat more recent (Stiglitz 2002 provides a useful overview). As Wilbur Schramm observes in a retrospective of the field, 'communication (is) a relationship built around the exchange of information' (Schramm 1983:5). However, the chapters in this volume reveal that communication through ICT has a number of unique characteristics which may shape these relationships. These include its capacity to pool, deconstruct, amplify, reconfigure, redistribute, store and curate information. In addition, the pivotal actors in this relationship who link ICT to beneficiaries may be individuals, institutions, corporates or governments or combinations of these. Further, ICT can be used in research to inform opinion or make decisions, comment and respond; in promotion/advocacy networking and organization and forms of identity or group formation; other forms of activism; sharing of information, opinions and preferences; and showing support, consensus or disagreement, scolding, abuse and flaming. On the other side, ICT can also be used for rule compliance and rule enforcement, surveillance, misinformation and propaganda, scolding and exposure; to give sermons; and to advance views of small connected groups over the other, most probably those of elites.

Understanding how these characteristics, dynamics and actors interact and the systems within which they operate is likely to be an area both for the application of existing approaches such as the actor-network theory and for the development of new concepts such as the ICT ecosystem. Of relevance here is the notion ICT 
renewing previous flagging communication relations that is used specifically around political communication by Fabro. This could apply to other dimensions as well such as intellectual curiosity, reading or art and suggests a role that ICT could play for greater engagement and agency promotion.

Closely related to this, a possible area of theory extension concerns the technical dimension of ICT and this might be a shortcoming of the current volume. This does not refer to better understanding of the technical component, but rather to understand the implications of different technical choices. These choices (and level of knowledge about the choice being made) may well shape later options and create a path dependency. Thus, there may be many arenas within which policy debate and conflict take place which typically include those that are political or bureaucratic. It may be necessary to recognize the importance of the technical arena in which design decisions are taken. As is shown in this volume, these design choices may influence the outcomes of interaction. One gap in our understanding that could address this gap concerns the origins of the ICT champions who make such decisions. This would include how such champions come about, how they survive or flourish and which champions do not succeed, including the reasons and the implications of this.

Digital poverty is an important theoretical advance discussed in Diga and May. However, as is the case with other alternatives proposed for the conceptualization of poverty, digital poverty does not necessarily map neatly onto more conventional forms of well-being. As an example, $15 \%$ of the nonpoor measured in a moneymetric approach are digitally poor. The reasons for this require further development and can be related to existing studies of subjective and structural poverty that have similar results.

Cruz and Sajo draw attention to the potentially subversive role of the Internet which simultaneously provides opportunities for exploitation and survival through cybersex. They also remind us of the potentially dark side of digital capitalism. Not all ICT is good, and equally not all deemed bad is necessarily bad for all. Some part of this consideration is ICT and financialization of capitalism and the consequences of this for development. A useful concept that they introduce is that of 'affective labour' and they raise the possible labour issues that might be associated with digital capitalism. The consequences of employment changes as a result of ICT, especially for those that are poor and marginalized, are an area of future investigation and should include considerations of the circumstances in which ICTs reduce or increase control over labour and perhaps provide freedom from drudgery or hazardous work.

Finally, future areas of research could make use of the typology of research proposed by Ordóñez to organize the ambitious task just provided. These are:

- Conceptual research which is blue-sky in nature

- Planning research, what to be done, in what sequence and where, establishing proof of mechanisms

- Instrumental research, testing new ideas, demonstrating proof of concept

- Action research

- Impact assessment/M\&E, attribution 
Any single research project may fall into multiple categories, and a study grounded in action research principles and methodologies may well have a conceptual component in which new ideas are explored or old ones reassessed.

Open Access This chapter is distributed under the terms of the Creative Commons Attribution Noncommercial License, which permits any noncommercial use, distribution, and reproduction in any medium, provided the original author(s) and source are credited.

\section{References}

Agre, P. E. (2002). Real-time politics: The internet and the political process. The Information Society, 18(5), 311-331.

Boaz, A., \& Pawson, R. (2005). The perilous road from evidence to policy: Five journeys compared. Journal of Social Policy, 34(2), 175-194.

Brewer, E., Demmer, M., Du, B., Ho, M., Kam, M., Nedevschi, S., \& Fall, K. (2005). The case for technology in developing regions. Computer, 38(6), 25-38.

Dutton, W. H. (2004). Social transformation in an information society: Rethinking access to you and the world. Paris: UNESCO. Retrieved from http://citeseerx.ist.psu.edu/viewdoc/download? doi=10.1.1.137.6534\&rep=rep1\&type=pdf. Accessed 16 Jan 2014.

Harris, R., \& Chib, A. (2012). Finding a path to influencing policy. In A. Chib \& R. W. Harris (Eds.), Linking research to practice: Strengthening ICT for development research capacity in Asia. Singapore: Institute of Southeast Asian Studies.

Heeks, R. (2010). Do information and communication technologies (ICTs) contribute to development? Journal of International Development, 22(5), 625-640.

Pawson, R. (2006). Evidence-based policy: A realist perspective. London: Sage.

Schramm, W. (1983). The unique perspective of communication: A retrospective view. Journal of Communication, 33(3), 6-17.

Stevenson, R. L. (1988). Communication, development, and the third world: The global politics of information. New York: Longman.

Stiglitz, J. E. (2002). Information and the change in the paradigm in economics. The American Economic Review, 92(3), 460-501.

Toyama, K. (2011, February). Technology as amplifier in international development. In Proceedings of the 2011 iConference (pp. 75-82). Seattle, USA: ACM. 